\title{
Fuzzy equational classes
}

\author{
Branka Budimirović and Vjekoslav Budimirović \\ Megatrend University \\ 11000 Belgrade, Serbia \\ e-mail: budimirovic@sbb.rs, vjekoslav@sbb.rs
}

\author{
Branimir Ššelja and Andreja Tepavčević \\ University of Novi Sad, \\ Trg Dositeja Obradovića 4, 21000 Novi Sad, Serbia \\ e-mail: seselja@dmi.uns.ac.rs, andreja@dmi.uns.ac.rs
}

\begin{abstract}
The paper deals with fuzzy equational classes. These are defined as classes of particular fuzzy algebras refereing to a fuzzy equality (which replaces the crisp one), closed with respect to fuzzy identities. In this fuzzy framework we introduce basic notions of universal algebra, (fuzzy) subalgebras, homomorphisms and direct products. Our main result is that every fuzzy equational class is closed under these three constructions, hence forming a fuzzy variety.
\end{abstract}

AMS Mathematics Subject Classification (2000): 03E72. Key words and phrases: fuzzy algebra, fuzzy identity, fuzzy equality, fuzzy homomorphism, fuzzy direct product, fuzzy equational class, fuzzy variety.

\section{INTRODUCTION}

Investigation of fuzzy algebraic structures has a long history within a fuzzy era. Most of the researchers have been dealing with particular algebras like fuzzy groups (starting with Rosenfeld 1971 [18], Das 1981 [8] and then followed by many others), fuzzy semigroups (see the monograph from 2003. by Malik, Mordeson and Kuroki [16]), and other structures with usually not more than two binary operations (semirings, rings, lattices and connected structures). These were further applied in other fields like fuzzy automata (e.g., the 2002. monograph by Mordeson and Malik [15]) and formal languages (e.g., Li, Pedrycz 2005, [14]). Notions from general and universal algebra have also been investigated in the fuzzy framework, like lattice valued algebras (starting by Di Nola and Gerla [11]), fuzzy congruences (Šešelja 1981 [19], Murali [17], and others). Investigating some basic notions in fuzzy mathematics, Demirci has been dealing with fuzzy functions and related notions ([9], [10]) and also Šostak, from the topological and categorical aspect ([28]). In the last decade of the XX century, also fuzzy homomorphisms were investigated (for fuzzy groups and generally, see Chakraborty and Khare, 1993 [7]). Researches of B. Šešelja and A. Tepavčević have also been addressed to fuzzy algebraic structures, as cited below. However, a systematic approach to fuzzy general algebra should be related to Bělohlávek and Vychodil papers (we point to his book together with Vychodil [2]).

In the present paper we deal with classes of fuzzy algebras which are defined within a fixed type of algebras. These fuzzy algebras are usual fuzzy subalgebras of crisp algebras of the same type, equipped with compatible fuzzy equalities, which generalize the ordinary crisp equality. The ordered structure of membership values is fixed for the whole class. If all fuzzy algebras in the class fulfill a set of particular formulas called fuzzy identities, then this class is called an equational class of fuzzy algebras. For the compatible fuzzy relations we use a special weak reflexivity; in addition, our approach to fuzzy identities, fuzzy functions and substructures differs from those existing in the literature, see also our paper [4].

Next we introduce basic algebraic constructions in the classes of fuzzy algebras. Namely, we define appropriate fuzzy subalgebras, fuzzy homomorphisms and fuzzy direct products. We prove that each equational class of fuzzy algebras is closed under these three constructions. As it is known, a class of the crisp algebras of the same type closed under the above constructions is called a variety. Therefore, here we prove that each fuzzy equational class is a fuzzy variety.

In dealing with fuzzy (lattice-valued) structures, we use our results obtained for the fuzzy groups and fuzzy algebras in general, but refereing to the crisp equalities ([20], [22], [23], [24], [25], [26], [5]). Our first attempt to deal with fuzzy identities is presented in [27]. What is directly used here are fuzzy algebras with fuzzy equalities, elaborated in [4]. In order to make the present material self contained, we list all the relevant definitions and properties from this article here in Preliminaries.

Finally, let us comment the structure of the membership values. In our investigations of universal algebra in the fuzzy framework, the structure of membership values should meet requirements for the relevant logical background. Therefore, we use a complete residuated lattice, though in the part of the research presented here it has some additional properties, by which it is a Heyting algebra.

\section{PRELIMINARIES}

\section{A. Crisp notions: algebras, equational classes, varieties}

First we list some notions and claims from the universal algebra; for more, refer to e.g., book [6].

For describing formally algebraic structures like groups, rings etc., we need a language or a type $\mathcal{L}$, which is a set $\mathcal{F}$ of functional symbols, together with a set of natural numbers with zero (arities) associated to these symbols. An algebra $\mathcal{A}=(A, F)$ of a type $\mathcal{L}$ consists of a nonempty set $A$, and a collection $F$ of (fundamental) operations on $A$. Each operation in $F$ corresponds to some symbol in the language; if it is $n$-ary, then its arity is $n$. Terms in the language $\mathcal{L}$ 
are usual regular expressions constructed by the variables and operational symbols (see [6] for the precise definition). An identity in $\mathcal{L}$ is a formula $t_{1}=t_{2}$, where $t_{1}, t_{2}$ are terms in the same language. A class of algebras of the same type, fulfilling a set of identities is an equational class. A subalgebra of $\mathcal{A}$ is an algebra of the same type, defined on a non-empty subset of $A$. A subset closed under fundamental operations is called a subuniverse of $\mathcal{A}$, which can also be the empty set. If $\mathcal{A}$ and $\mathcal{B}$ are algebras of the same type, then the function $h: A \rightarrow B$ compatible with the fundamental operations in the sense that for an $n$-ary $f \in F$, and $x_{1}, \ldots, x_{n} \in A, h\left(f\left(x_{1}, \ldots, x_{n}\right)\right)=$ $f\left(h\left(x_{1}\right), \ldots, h\left(x_{n}\right)\right)$ is a homomorphism of $\mathcal{A}$ into $\mathcal{B}$. The set of images $h(A)$ under $h$ is a subalgebra of $\mathcal{B}$, a homomorphic image of $\mathcal{A}$. For a family $\left\{\mathcal{A}_{i} \mid i \in I\right\}$ of algebras of the same type, $\Pi_{i \in I} \mathcal{A}_{i}$ is their direct product, an algebra of the same type with operations defined componentwise. A class of algebras of the same type is a variety if it is closed under subalgebras, homomorphic images and direct products. By the well known Birkhoff's theorem, an equational class is a variety and vice versa. An equivalence relation $\rho$ on $\mathcal{A}$ which is compatible with respect to all fundamental operations $\left(x_{i} \rho y_{i}, i=1, \ldots, n\right.$ imply $\left.f\left(x_{1}, \ldots, x_{n}\right) \rho f\left(y_{1}, \ldots, y_{n}\right)\right)$ is a congruence relation on $\mathcal{A}$. If $\rho$ is a congruence on $\mathcal{A}$, then $A / \rho$ is the quotient algebra, where the underlying set consists of congruence classes under $\rho$, and operations are defined over representatives. $A / \rho$ is a homomorphic image of $\mathcal{A}$ under $x \mapsto[x]_{\rho}$, where $[x]_{\rho}$ is the congruence class to which $x$ belongs.

\section{B. Fuzzy notions}

First we introduce the structure of membership values that we use in this research. Definitions follow, and more details can be found in [1].

A complete residuated lattice $(L, \wedge, \vee, \otimes, \rightarrow, 0,1)$ is an algebraic structure in which:

$(L, \wedge, \vee, 0,1)$ is a complete lattice, i.e., a partially ordered set whose ordering is denoted by $\leqslant$, and in which for every subset there is the greatest lower bound, infimum, meet, and the least upper bound, supremum, join; 0 is the least element (the bottom) and 1 is the greatest element (the top);

$(L, \otimes, 1)$ is a commutative monoid with the unit 1 ;

operations $\otimes$ (multiplication) and $\rightarrow$ (residuum) form an adjoint pair, meaning that for all $x, y, z \in L$

$x \otimes y \leqslant z$ if and only if $x \leqslant y \rightarrow z$.

A residuated lattice in which multiplication $(\otimes)$ coincides with meet $(\wedge)$ is a Heyting algebra.

Throughout the paper, $L$ is supposed to be the above introduced ordered structure and it is used as a set of membership values for all the fuzzy objects.

Next we list some relevant notions concerning fuzzy relations. Some of these are known and some are introduced in our paper [4].
A fuzzy set $\mu$ on a nonempty set $A$ is a function $\mu: A \rightarrow L$. Consequently, a mapping $\rho: A^{2} \rightarrow L$ is a fuzzy relation on A.

What we use here are the fuzzy relations on fuzzy sets, defined as follows. If $\mu: A \rightarrow L$ is a fuzzy set on a nonempty set $A$, then a fuzzy relation $\rho: A^{2} \rightarrow L$ on $A$ is said to be a fuzzy relation on $\mu$ if for all $x, y \in A$

$$
\rho(x, y) \leqslant \mu(x) \otimes \mu(y) .
$$

A fuzzy relation $\rho$ on fuzzy set $\mu$ is reflexive if for all $x, y \in$ A,

$$
\rho(x, x)=\mu(x) .
$$

The following is obvious.

Lemma 1: If $\rho$ is a reflexive fuzzy relation on a fuzzy set $\mu$ on $A$, then for every $x, y \in A$,

$$
\rho(x, x) \geqslant \rho(x, y) \text { and } \rho(x, x) \geqslant \rho(y, x) .
$$

A fuzzy relation on a fuzzy set $\mu$ on $A$ is symmetric and transitive if it fulfills these conditions as a fuzzy relation on crisp domain $A$ :

$\rho$ is symmetric if $\rho(x, y)=\rho(y, x)$ for all $x, y \in A$;

$\rho$ is transitive if $\rho(x, y) \geqslant \rho(x, z) \otimes \rho(z, y)$ for all $x, y, z \in A$.

A reflexive, symmetric and transitive relation $\rho$ on a fuzzy set $\mu$ is a fuzzy equivalence on $\mu$.

A fuzzy equivalence relation $\rho$ on $\mu$, fulfilling for all $x, y \in$ $A, x \neq y$, :

$$
\text { if } \rho(x, x) \neq 0 \text {, then } \rho(x, x)>\rho(x, y) \text {, }
$$

is called a fuzzy equality relation on a fuzzy set $\mu$.

Let $\mathcal{A}=(A, F)$ be a crisp algebra. As it is known, a fuzzy subalgebra of $\mathcal{A}$ is any mapping $\mu: A \rightarrow L$ fulfilling the following:

For any operation $f$ from $F$ with the arity greater than 0 , $f: A^{n} \rightarrow A, n \in \mathbb{N}$, and all $x_{1}, \ldots, x_{n} \in A$, we have that

$$
\bigotimes_{i=1}^{n} \mu\left(x_{i}\right) \leqslant \mu\left(f\left(x_{1}, \ldots, x_{n}\right)\right) .
$$

For a nullary operation (constant) $c \in F$, we require that

$$
\mu(c)=1,
$$

where 1 is the greatest (the top) element in $L$.

A fuzzy relation $\rho: A^{2} \rightarrow L$ on a fuzzy subalgebra $\mu: A \rightarrow$ $L$ of $\mathcal{A}=(A, F)$ is said to be compatible with the operations, if it is compatible as a fuzzy relation on $\mathcal{A}$, i.e., if for every $n$-ary operation $f \in F$ and for all $x_{1}, \ldots, x_{n}, y_{1}, \ldots, y_{n} \in A$

$$
\rho\left(f\left(x_{1}, \ldots, x_{n}\right), f\left(y_{1}, \ldots, y_{n}\right)\right) \geqslant \bigotimes_{i=1}^{n} \rho\left(x_{i}, y_{i}\right) .
$$


In particular, for $n=1, \rho\left(f\left(x_{1}\right), f\left(y_{1}\right)\right) \geqslant \rho\left(x_{1}, y_{1}\right)$.

A compatible fuzzy equivalence on a fuzzy subalgebra $\mu$ of $\mathcal{A}$ is a fuzzy congruence on $\mu$. Obviously, particular fuzzy congruences on $\mu$ are compatible fuzzy equalities on this fuzzy subalgebra.

\section{RESULTS}

First we introduce a new concept of fuzzy algebras, and then we generalize the basic relevant notions: subalgebras, homomorphisms and direct products.

\section{A. Fuzzy algebra, identity, equational class}

Let $\mathcal{A}=\left(A, F_{A}\right)$ be an algebra and let $L$ be a structure of membership values, as introduced above. Further, let $\mu_{A}$ : $A \rightarrow L$ be a fuzzy subalgebra of $\mathcal{A}$ and $E_{A}: A^{2} \rightarrow L$ a compatible fuzzy equality on $\mu_{A}$. Then we say that the fourtuple $\overline{\mathcal{A}}=\left(\mathcal{A}, \mu_{A}, E_{A}, L\right)$ is a fuzzy algebra of the type $(F, \sigma)$.

In other words, a fuzzy algebra is a fuzzy L-valued subalgebra of a given crisp algebra, endowed with a compatible fuzzy equality. That is why all relevant parameters are explicitly listed in the defining four-tuple.

Remark 1: If we take $\mu_{A}: A \rightarrow L$ to be a characteristic function, then the above definition gives an algebra with fuzzy equality, [3] (actually a subalgebra of $\mathcal{A}$ ). If in addition $E_{A}$ is a crisp equality, then a classical subalgebra of $\mathcal{A}$ is obtained.

Clearly, the above definition implies that two given fuzzy algebras $\overline{\mathcal{A}}=\left(\mathcal{A}, \mu_{A}, E_{A}, L\right)$ and $\overline{\mathcal{B}}=\left(\mathcal{B}, \mu_{B}, E_{B}, L\right)$ coincide if $\mathcal{A}=\mathcal{B}, \mu_{A}=\mu_{B}, E_{A}=E_{B}$ and both refer to the same lattice $L$.

As defined in [4], a fuzzy identity of the type $(F, \sigma)$ over a set of variables $X$ is the expression $E\left(t_{1}, t_{2}\right)$, where $t_{1}\left(x_{1}, \ldots, x_{n}\right), t_{2}\left(x_{1}, \ldots, x_{n}\right)$, briefly $t_{1}, t_{2}$ belong to the set $T(X)$ of terms over $X$ and both have at most $n$ variables.

We say that a fuzzy algebra $\overline{\mathcal{A}}=\left(\mathcal{A}, \mu_{A}, E_{A}, L\right)$ satisfies a fuzzy identity $E\left(t_{1}, t_{2}\right)$ (or that this identity holds on $\overline{\mathcal{A}}$ ), if for all $x_{1}, \ldots, x_{n} \in A$

$$
\bigotimes_{i=1}^{n} \mu_{A}\left(x_{i}\right) \leqslant E_{A}\left(t_{1}, t_{2}\right) .
$$

Remark 2: In paper [4] we proved that for every fuzzy identity $E\left(t_{1}, t_{2}\right)$ on a fuzzy subalgebra $\mu$ of a crisp algebra $\mathcal{A}$, there is the smallest compatible fuzzy equality $E_{m}$, such that $E\left(t_{1}, t_{2}\right)$ holds on $\mu$. The approach in the present paper is slightly different, since here we deal with a fuzzy algebra, which is by definition a fuzzy subalgebra $\mu$, together with a compatible fuzzy equality on it.

Let $\Sigma$ be a set of fuzzy identities of the type $(F, \sigma)$ and let $L$ be fixed. Then all the fuzzy algebras $\overline{\mathcal{A}}=\left(\mathcal{A}, \mu_{A}, E_{A}, L\right)$ of this type satisfying all identities in $\Sigma$ form an equational class $\mathfrak{M}$ of fuzzy algebras.

\section{B. Fuzzy subalgebra of a fuzzy algebra}

Here we start with a fuzzy algebra $\overline{\mathcal{A}}=\left(\mathcal{A}, \mu_{A}, E_{A}, L\right)$ and identify particular fuzzy subalgebras of $\mathcal{A}$ as fuzzy subalgebras of $\overline{\mathcal{A}}$.

The next theorem formalizes our intuition and enables the definition that follows.

Theorem 1: Let $\overline{\mathcal{A}}=\left(\mathcal{A}, \mu_{A}, E_{A}, L\right)$ be a fuzzy algebra and $\mu_{B}: A \rightarrow L$ a fuzzy subalgebra of $\mathcal{A}$, fulfilling the following conditions:

1) $\mu_{B}(x) \leqslant \mu_{A}(x)$ for all $x \in A$.

2) If $x$ and $y$ are distinct elements of $A$ and $\mu_{B}(x)>0$, then $E_{A}(x, y)<\mu_{B}(x)$.

3) $\mu_{B}(c)=\mu_{A}(c)$, for any constant $c$ in the language.

Then, a fuzzy relation $E_{B}$ on $\mu_{B}$ given by

$$
E_{B}(x, y):=E_{A}(x, y) \otimes \mu_{B}(x) \otimes \mu_{B}(y),
$$

is a compatible fuzzy equality on $\mu_{B}$.

Now we are ready for the following definition.

Let $\overline{\mathcal{A}}=\left(\mathcal{A}, \mu_{A}, E_{A}, L\right)$ be a fuzzy algebra and $\mu_{B}: A \rightarrow$ $L$ a fuzzy subalgebra of $\mathcal{A}$, fulfilling the following:

1) $\mu_{B}(x) \leqslant \mu_{A}(x)$ for all $x \in A$.

2) If $x$ and $y$ are distinct elements from $A$ and if $\mu_{B}(x)>$ 0 , then $E_{A}(x, y)<\mu_{B}(x)$.

3) $\mu_{B}(c)=\mu_{A}(c)$, for any constant $c$.

4) $E_{B}(x, y):=E_{A}(x, y) \otimes \mu_{B}(x) \otimes \mu_{B}(y)$.

Then we say that the fuzzy algebra $\overline{\mathcal{B}}=\left(\mathcal{A}, \mu_{B}, E_{B}, L\right)$ is a (fuzzy) subalgebra of the fuzzy algebra $\overline{\mathcal{A}}$.

Theorem 2: Let $\mathfrak{M}$ be an equational class of fuzzy algebras and let $\overline{\mathcal{A}} \in \mathfrak{M}$ where $\overline{\mathcal{A}}=\left(\mathcal{A}, \mu_{A}, E_{A}, L\right)$. If $\overline{\mathcal{B}}=$ $\left(\mathcal{A}, \mu_{B}, E_{B}, L\right)$ is a fuzzy subalgebra of $\overline{\mathcal{A}}$, then also $\overline{\mathcal{B}} \in \mathfrak{M}$.

Observe that by the definition of a fuzzy subalgebra it follows that

$$
u_{A}\left(x_{1}, \ldots, x_{n}\right)=u_{B}\left(x_{1}, \ldots, x_{n}\right)
$$

for every term $u\left(x_{1}, \ldots, x_{n}\right)$.

\section{Fuzzy homomorphism}

Let $\overline{\mathcal{A}}=\left(\mathcal{A}, \mu_{A}, E_{A}, L\right)$ and $\overline{\mathcal{B}}=\left(\mathcal{B}, \mu_{B}, E_{B}, L\right)$ be fuzzy algebras of the same type. We say that $f: A \rightarrow B$ is a fuzzy mapping of $\overline{\mathcal{A}}$ into $\overline{\mathcal{B}}$ if the following conditions hold:

1. $(\forall a \in A) \mu_{B}(f(a)) \geqslant \mu_{A}(a)$

2. Let $t_{1}\left(x_{1}, \ldots, x_{n}\right), t_{2}\left(x_{1}, \ldots, x_{n}\right)$ be terms in the language of $\mathcal{A}$, let $t_{1}^{A}, t_{2}^{A}$ be the corresponding term operations and $a_{1}, \ldots, a_{n}$ elements from $A$. 


$$
\text { If } \begin{array}{ll} 
& E_{A}\left(t_{1}^{A}\left(a_{1}, \ldots, a_{n}\right), t_{2}^{A}\left(a_{1}, \ldots, a_{n}\right)\right) \geqslant \bigotimes_{i=1}^{n} \mu_{A}\left(a_{i}\right), \\
\text { then } \quad & E_{B}\left(f\left(t_{1}^{A}\left(a_{1}, \ldots, a_{n}\right)\right), f\left(t_{2}^{A}\left(a_{1}, \ldots, a_{n}\right)\right)\right) \geqslant \\
& \mu_{B}\left(f\left(t_{1}^{A}\left(a_{1}, \ldots, a_{n}\right)\right)\right) \otimes \mu_{B}\left(f\left(t_{2}^{A}\left(a_{1}, \ldots, a_{n}\right)\right)\right) .
\end{array}
$$

Remark 3: A fuzzy mapping defined here differs from the one defined by Demirci in [9], [10]. In the cited articles the domain is a crisp set equipped with a fuzzy equality and a fuzzy mapping is defined as a particular fuzzy binary relation. In our approach, both the domain and the co-domain are fuzzy sets, while the mapping is a special ordinary function.

Theorem 3: Let $\overline{\mathcal{A}}=\left(A, \mu_{A}, E_{A}, L\right), \overline{\mathcal{B}}=\left(B, \mu_{B}, E_{B}, L\right)$ and $\overline{\mathcal{C}}=\left(\mathcal{C}, \mu_{C}, E_{C}, L\right)$ be fuzzy algebras of the same type and $f: A \rightarrow B, g: B \rightarrow C$ fuzzy mappings. Then also their composition $f \circ g: A \rightarrow C$ is a fuzzy mapping.

Let $\overline{\mathcal{A}}=\left(\mathcal{A}, \mu_{A}, E_{A}, L\right)$ and $\overline{\mathcal{B}}=\left(\mathcal{B}, \mu_{B}, E_{B}, L\right)$ be fuzzy algebras of the same type. We say that the fuzzy mapping $h: A \rightarrow B$ is a fuzzy homomorphism of the fuzzy algebra $\overline{\mathcal{A}}$ into the fuzzy algebra $\overline{\mathcal{B}}$ if the following holds:

1) For each $n$-ary operation $f_{A}$ and for all $a_{1}, \ldots, a_{n} \in A$, $h\left(f_{A}\left(a_{1}, \ldots, a_{n}\right)\right)=f_{B}\left(h\left(a_{1}\right), \ldots, h\left(a_{n}\right)\right)$.

2) $h\left(c_{A}\right)=c_{B}$, for every nullary operation $c$ in the language, $c_{A}$ and $c_{B}$ being the corresponding constants in $\mathcal{A}$ and $\mathcal{B}$ respectively.

Obviously, a fuzzy homomorphism is a fuzzy mapping which is also a homomorphism.

In the following proposition we introduce a particular fuzzy subalgebra, which we need in order to locate a homomorphic image of a fuzzy homomorphism.

Proposition 1: Let $\overline{\mathcal{A}}=\left(\mathcal{A}, \mu_{A}, E_{A}, L\right)$ be a fuzzy algebra and $\mathcal{B}=\left(B, F_{B}\right)$ a crisp subalgebra of $\mathcal{A}$. If

$$
\begin{gathered}
\mu_{B}(x):=\left\{\begin{aligned}
\mu_{A}(x), & x \in B \\
0, & \text { else }
\end{aligned}\right. \text { and } \\
E_{B}(x, y):=E_{A}(x, y) \otimes \mu_{B}(x) \otimes \mu_{B}(y),
\end{gathered}
$$

then $\overline{\mathcal{B}}=\left(\mathcal{A}, \mu_{B}, E_{B}, L\right)$ is a fuzzy subalgebra of $\overline{\mathcal{A}}$.

Theorem 4: Let $\overline{\mathcal{A}}=\left(\mathcal{A}, \mu_{A}, E_{A}, L\right)$ and $\overline{\mathcal{B}}=$ $\left(\mathcal{B}, \mu_{B}, E_{B}, L\right)$ be fuzzy algebras and $h: A \rightarrow B$ a fuzzy homomorphism. Define $\overline{\mathcal{D}}=\left(\mathcal{B}, \mu_{D}, E_{D}, L\right)$, where

$$
\mu_{D}(d):=\left\{\begin{aligned}
\mu_{B}(d), & d \in D=h(A) \\
0, & \text { otherwise }
\end{aligned}\right.
$$

and

$$
E_{D}(x, y)=E_{B}(x, y) \otimes \mu_{D}(x) \otimes \mu_{D}(y) .
$$

Then, $\overline{\mathcal{D}}$ is a fuzzy subalgebra of fuzzy algebra $\overline{\mathcal{B}}$.
Fuzzy subalgebra $\overline{\mathcal{D}}=\left(\mathcal{B}, \mu_{D}, E_{D}, L\right)$ of $\overline{\mathcal{B}}$, introduced in Theorem 4 , is said to be the homomorphic image of fuzzy algebra $\overline{\mathcal{A}}$.

Theorem 5: Let $\overline{\mathcal{A}}=\left(\mathcal{A}, \mu_{A}, E_{A}, L\right)$ and $\overline{\mathcal{B}}=$ $\left(\mathcal{B}, \mu_{B}, E_{B}, L\right)$ be fuzzy algebras and $h: A \rightarrow B$ a fuzzy homomorphism. If $F\left(x_{1}, \ldots, x_{n}\right)$ is a term in the same language and $F^{A}, F^{B}$ the corresponding term operations in $\mathcal{A}$ and $\mathcal{B}$ respectively, then $h$ is a fuzzy homomorphism of fuzzy algebra $\left(\overline{\mathcal{A}}, F^{A}\right)$ into fuzzy algebra $\left(\overline{\mathcal{B}}, F^{B}\right)$.

Proof: Straightforwardly, by the corresponding theorem in the classical general algebra.

We are now ready to prove that the equational classes of fuzzy algebras are closed under the homomorphic images.

Theorem 6: Let $\mathfrak{M}$ be an equational class of fuzzy algebras. If $\overline{\mathcal{A}} \in \mathfrak{M}$ and $\overline{\mathcal{D}}$ is a homomorphic image of $\overline{\mathcal{A}}$, then also $\overline{\mathcal{D}} \in \mathfrak{M}$.

\section{Direct product of fuzzy algebras}

Here we deal with the third algebraic construction in the fuzzy framework, namely with the fuzzy direct products.

Theorem 7: Let $\left\{\overline{\mathcal{A}}_{i}=\left(\mathcal{A}_{i}, \mu_{i}, E_{A_{i}}\right), L \mid i \in I\right\}$ be a family of fuzzy algebras of the same type, $\mathcal{A}=\prod_{i \in I} \mathcal{A}_{i}$ the direct product of algebras $\mathcal{A}_{i}$ and let the following holds for all $g_{1}, g_{2} \in \prod_{i \in I} \mathcal{A}_{i}, g_{1} \neq g_{2}$ :

$$
\begin{aligned}
& \text { If } \bigotimes_{i \in I} \mu_{i}\left(g_{1}(i)\right) \neq 0 \text {, then } \\
& \qquad \bigotimes_{i \in I} E_{A_{i}}\left(g_{1}(i), g_{2}(i)\right) \neq \bigotimes_{i \in I} \mu_{i}\left(g_{1}(i)\right) .
\end{aligned}
$$

Then the following holds: If

1) $\mu(g):=\bigotimes_{i \in I} \mu_{i}(g(i)), g \in \prod_{i \in I} A_{i}$ and

2) $E_{A}\left(g_{1}, g_{2}\right):=\bigotimes_{i \in I} E_{A_{i}}\left(g_{1}(i), g_{2}(i)\right) ; g_{1}, g_{2} \in \prod_{i \in I} A_{i}$,

then $\overline{\mathcal{A}}=\prod_{i \in I} \overline{\mathcal{A}}_{i}:=\left(\mathcal{A}, \mu, E_{A}, L\right)$ is a fuzzy algebra.

The fuzzy algebra $\overline{\mathcal{A}}:=\left(\mathcal{A}, \mu, E_{A}, L\right)$ introduced in Theorem 7 is said to be the direct product of fuzzy algebras $\overline{\mathcal{A}}_{i}, i \in I$.

Remark 4: The technical condition

$$
\bigotimes_{i \in I} E_{A_{i}}\left(g_{1}(i), g_{2}(i)\right) \neq \bigotimes_{i \in I}\left(\mu_{i}\left(g_{1}(i)\right)\right)
$$

in the definition of the fuzzy direct product is needed in the proof of Theorem 7 which is not provided here due to lack of space.

Observe that the corresponding condition for the crisp algebras is trivially fulfilled.

Theorem 8: If a fuzzy identity

$E\left(u\left(x_{1}, \ldots, x_{n}\right), v\left(x_{1}, \ldots, x_{n}\right)\right)$ holds in all fuzzy algebras 
$\overline{\mathcal{A}}_{i}, i \in I$ of a fixed type, then also this fuzzy identity holds in their product $\overline{\mathcal{A}}=\prod_{i \in I} \overline{\mathcal{A}}_{i}$.

Now we formulate the main result, i.e., we prove that any fuzzy equational class is closed under fuzzy subalgebras, homomorphic images and products.

Theorem 9: Let $\mathfrak{M}$ be an equational class of fuzzy algebras. Then the following hold:

1) If $\overline{\mathcal{A}} \in \mathfrak{M}$, and $\overline{\mathcal{B}}$ is a fuzzy subalgebra of $\overline{\mathcal{A}}$, then $\overline{\mathcal{B}} \in$ $\mathfrak{M}$.

2) If $\overline{\mathcal{A}} \in \mathfrak{M}$, and $\overline{\mathcal{D}}$ is a homomorphic image of $\overline{\mathcal{A}}$, then $\overline{\mathcal{D}} \in \mathfrak{M}$.

3) If for every $i \in I, \quad \overline{\mathcal{A}}_{i}$ belongs to $\mathfrak{M}$, then also $\prod_{i \in I} \overline{\mathcal{A}}_{i} \in$ $\mathfrak{M}$.

Proof: Straightforward by Theorems 2, 6, 8 .

In order to formulate the above result appropriately in terms of general algebra, let us define the following notion. For a fixed language and a complete residuated lattice $L$, a class $\mathcal{V}$ of fuzzy algebras $\overline{\mathcal{A}}=\left(\mathcal{A}, \mu_{A}, E_{A}, L\right)$ closed under fuzzy subalgebras, fuzzy homomorphic images and fuzzy direct products is a fuzzy variety.

In terms of fuzzy varieties, Theorem 2 can be formulated as follows.

Corollary 1: Every fuzzy equational class is a fuzzy variety.

Theorem 9 (1) is a one way fuzzy version of the famous Birkhoff Theorem in universal algebra (see Preliminaries). Under the present conditions, the converse is not true. E.g., the class of crisp commutative groupoids with the usual equality is closed under formation of (fuzzy) subalgebras, homomorphisms and products, as they are defined here. However, the corresponding equational class defined by the fuzzy identity $E(x y, y x)$ is wider, as shown by the following example (taken from [4]).

Example Let $G$ be a four-element groupoid given by the Table 1, and $L$ a 16-element Boolean lattice (Figure 1). Boolean lattice is residuated and multiplication coincides with meet.

\begin{tabular}{l|llll}
$\cdot$ & $a$ & $b$ & $c$ & $d$ \\
\hline$a$ & $b$ & $b$ & $c$ & $c$ \\
$b$ & $b$ & $a$ & $d$ & $d$ \\
$c$ & $c$ & $c$ & $d$ & $d$ \\
$d$ & $d$ & $d$ & $d$ & $c$
\end{tabular}

Table 1: Groupoid $G$

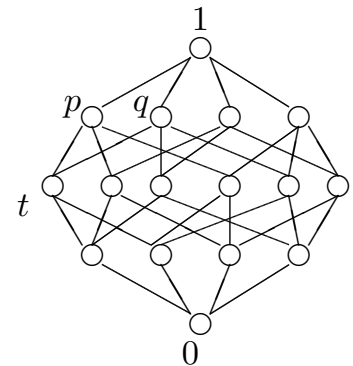

Figure 1: Lattice $L$

\begin{tabular}{c|cccc}
$E_{m}$ & $a$ & $b$ & $c$ & $d$ \\
\hline$a$ & $p$ & 0 & 0 & 0 \\
$b$ & 0 & $p$ & 0 & 0 \\
$c$ & 0 & 0 & $q$ & $t$ \\
$d$ & 0 & 0 & $t$ & $q$
\end{tabular}

Table 2: Fuzzy equality $E_{m}$

Consider the fuzzy groupoid $(G, \mu, E)$, where $\mu: G \rightarrow L$ is given by:

$$
\mu(x)=\left(\begin{array}{llll}
a & b & c & d \\
p & p & q & q
\end{array}\right) .
$$

If $E$ is a fuzzy equality on $G$ presented by the Table 2, then $(G, \mu, E, L)$ fulfils the fuzzy identity $E(x \cdot y, y \cdot x)$. Though, this fuzzy commutative groupoid could not be obtained by $H, S, P$ operators applied on crisp commutative groupoids.

\section{CONCLUSION}

In the present research we are focused on fuzzy identities as a new fuzzy notion generalizing crisp identities and we apply these to fuzzy algebras. This is a natural way to define fuzzy equational classes. It is obvious that one way round results, starting with fuzzy equational classes and proving that these are fuzzy varieties should be investigated also in the opposite direction. In order to fulfil this task we intend to further investigate fuzzy algebras. These should satisfy additional conditions, so that when closed under (fuzzy) subalgebras, homomorphisms and products, they form an equational class.

\section{ACKNOWLEDGEMENT}

The research of the second author is supported by the Ministry of Education and Science of the Republic of Serbia, Grant No. 44006.

The research of the last two authors are supported by the Ministry of Education and Science of the Republic of Serbia, Grant No. 174013 and by the Provincial Secretariat for Science and Technological Development, Autonomous Province of Vojvodina, grant "Ordered structures and applications".

\section{REFERENCES}

[1] R. Bělohlávek, Fuzzy Relational Systems: Foundations and Principles, Kluwer Academic/Plenum Publishers, New York, 2002.

[2] R. Bělohlávek, V. Vychodil, Fuzzy Equational Logic, Studies in Fuzziness and Soft Computing, Volume 186, Springer 2005.

[3] R. Bělohlávek, V. Vychodil, Algebras with fuzzy equalities, Fuzzy Sets and Systems 157 (2006) 161-201. 
[4] B. Budimirović, V. Budimirović, B. Šešelja, A. Tepavčević, Compatible fuzzy equalities and fuzzy identities, submitted.

[5] B. Budimirović, V. Budimirović, A. Tepavčević, Fuzzy $\varepsilon$-Subgroups, Information Sciences 180 (2010) 4006-4014.

[6] S. Burris, H.P. Sankappanavar, A Course in Universal Algebra, SpringerVerlag, N.Y. 1981

[7] A.B. Chakraborty, S.S. Khare, Fuzzy homomorphism and algebraic structures, Fuzzy Sets and Systems 59 (1993) 211-221.

[8] P.S. Das, Fuzzy groups and level subgroups, J. Math. Anal. Appl. 84 (1981) 264-269.

[9] M. Demirci, Vague Groups, J. Math. Anal. Appl. 230 (1999) 142-156.

[10] M. Demirci, Foundations of fuzzy functions and vague algebra based on many-valued equivalence relations part I: fuzzy functions and their applications, part II: vague algebraic notions, part III: constructions of vague algebraic notions and vague arithmetic operations, Int. J. General Systems 32 (2003) 123-155, 157-175, 177-201.

[11] A. Di Nola, G. Gerla, Lattice valued algebras, Stochastica 11 (1987) 137-150.

[12] U. Höhle, Quotients with respect to similarity relations, Fuzzy Sets and Systems 27 (1988) 31-44.

[13] G. Klir, B. Yuan, Fuzzy sets and fuzzy logic, Prentice Hall P T R, New Jersey, 1995.

[14] Y.M. Li, W. Pedrycz, Fuzzy finite automata and fuzzy regular expressions with membership values in lattice ordered monoids, Fuzzy Sets and Systems 156 (2005) 68-92.

[15] J.N. Mordeson and D.S. Malik, Fuzzy Automata and Languages Theory and Applications. Computational Mathematics Series. Chapman \& Hall/CRC, 2002.

[16] J.N. Malik, D.S. Mordeson, N. Kuroki, Fuzzy Semigroups, Springer, 2003.

[17] V. Murali, Fuzzy congruence relations, Fuzzy Sets and Systems 41 (1991) 359-369.

[18] A. Rosenfeld, Fuzzy Groups, J. Math. Anal. Appl. 36 (1971), 512-517.

[19] B. Šešelja, Characterization of fuzzy equivalence relations and of fuzzy congruence relations on algebras, Review of Research Faculty of Science - University of Novi Sad 11 (1981) 153-160.

[20] B. Šešelja, Homomorphisms of poset-valued algebras Fuzzy Sets and Systems 121 (2001) 333-340.

[21] B. Šešelja, A. Tepavčević, Weak Congruences in Universal Algebras, Institute of Mathematics, Novi Sad 2001.

[22] B. Sešelja, A. Tepavčević, Fuzzy Boolean algebras, Automated Reasoning, IFIP Transactions A-19, (1992) 83-88.

[23] B. Šešelja, A. Tepavčević, Partially Ordered and Relational Valued Algebras and Congruences, Review of Research, Faculty of Science, Mathematical Series 23 (1993) 273-287.

[24] B. Šešelja, A. Tepavčević, On Generalizations of Fuzzy Algebras and Congruences, Fuzzy Sets and Systems 65 (1994) 85-94.

[25] B. Sešelja, A. Tepavčević, Fuzzy groups and collections of subgroups, Fuzzy Sets and Systems 83 (1996) 85-91.

[26] B. Sešelja, A. Tepavčević, A note on fuzzy groups, YUJOR 7 (1997) 49-54.

[27] B. Šešelja, A. Tepavčević, Fuzzy Identities, Proc. of the 2009 IEEE International Conference on Fuzzy Systems 1660-1664.

[28] A.P. Šostak, On a fuzzy topological structure, Frolík, Z., Soucek, V. and Vinárek, J. (eds.): Proceedings of the 13th Winter School on Abstract Analysis. Section of Topology, Palermo, 1985 89-103. 